\title{
Research on Gearbox Fault Detection and Diagnosis Based on Improved Spectral Kurtosis Algorithm
}

\author{
Lijun $\mathrm{Cao}^{1, *}$, Yanqin Zhao ${ }^{2}$, Guibo $\mathrm{Yu}^{1}$, Shuxiao $\mathrm{Chen}^{3}$ and Xujun $\mathrm{Su}^{1}$ \\ ${ }^{1}$ Shijiazhuang Mechanical Engineering College, Shijiazhuang, Hebei, 050003, P.R. China \\ ${ }^{2}$ Department of Defense Mobilization, Beijing, 100034, P.R. China \\ ${ }^{3}$ Shijiazhuang Mechanical Engineering College, Shijiazhuang, Hebei, 050084, P.R. China \\ ${ }^{*}$ Corresponding author
}

\begin{abstract}
A gearbox fault detection and diagnosis test table of wheeled armored vehicles is designed and established. Typical faults and vibration experiments can be preset. Aiming to the $d$ that traditional spectral kurtosis algorithm cannot be applied to gearbox fault signal feature extraction under strong noise interference, the minimum entropy deconvolution theory is adopted, and a new kind of gearbox fault diagnosis method based on MED and FSK is proposed, which realizes the single fault diagnosis of gears and bearings at different speed conditions, and also achieves good result for composite fault diagnosis of rolling bearing inner and outer rings. Compared with the traditional method of wavelet analysis and EMD, the proposed method for gearbox vibration signal has better noise reduction result.
\end{abstract}

Keywords-spectral kurtosis; minimum entropy deconvolution; gearbox; fault detection; fault diagnosis

\section{INTRODUCTION}

Gearbox is the important transmission device of mechanical equipments. It has complicated internal structure and high assembly accuracy. Because of the formidable working conditions, the fault rate of gearbox is high and the corresponding fault influence is enormous. Spectral kurtosis is a kind of signal transaction method to detect the ballistic part in signal. Its basic principle is computing the durtosis value of every spectral line and reflecting the transient ballistic corresponding to different durtosis value. Because of the rather large interference of working condition to gearbox's vibration signals, the signal extraction effectiveness of traditional spectral kurtosis algorithm for low SNR signal is not obvious $^{[1][2]}$. In this paper, the minimum entropy deconvolution theory is adopted and a new kind of gearbox fault detection and diagnosis method based on minimum entropy deconvolution and rapid spectral kurtosis algorithm is put forward. It retains the advantages of traditional spectral kurtosis algorithm to extract the signal characteristics by adaptive band-pass filter parameter selection. The credibility is verified by gearbox's single fault diagnosis test and bearing inward-outward ring compound fault diagnosis test.

\section{GEARBox FAULt DeteCtion AND DiagnOSIS TEST}

In the fault diagnosis process of gearbox, the signals of normal state are relative easy to collect. But in practical engineering, there are many types of gearbox's fault. Especially, weak faults after complex transmission require optimal measuring stations for sensors. Gearbox usually adopt sealed structure and is difficult to install sensors and collect fault signals. To collect sufficient fault signals for fault detection diagnosis, a gearbox fault diagnosis test table is established. Typical faults can be located based on the test table to explore and verify the corresponding gearbox fault diagnosis method.

\section{A. Composition of Test Table}

The test table of gearbox fault detection and diagnosis is composed of electromagnetic variable-speed motor, secondlevel decelerator, clutch, sensors, direct-current power, magnetic-particle brake and signal collection instrument. The test table composition is shown as Figure I.

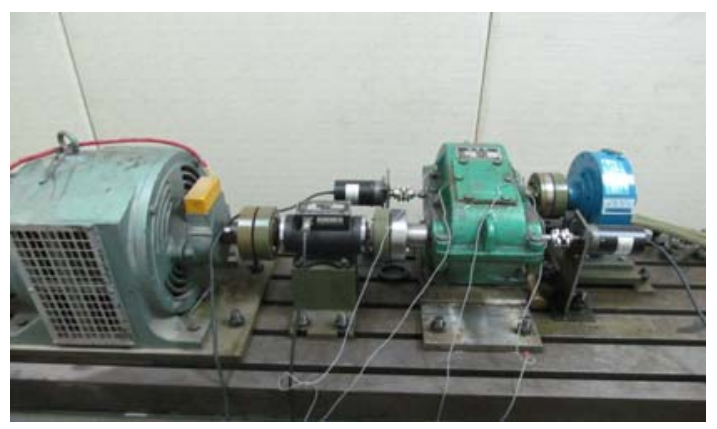

FIGURE I. THE TEST TABLE COMPOSITION OF GEARBOX FAULT DETECTION AND DIAGNOSIS

TABLE I. COMPOSITION OF GEARBOX TEST TABLE

\begin{tabular}{|c|c|c|}
\hline Name & Type & Number \\
\hline Electromagnetic variable-speed motor & YCT180-4A & 1 \\
\hline Gearbox & 200 & 1 \\
\hline Clutch & & 2 \\
\hline Magnetic-particle brake & FZJ-5 & 1 \\
\hline Direct-current power & WLY-1A & 1 \\
\hline Rotation speed and rotation torque & JN338 & 1 \\
\hline Vibration acceleration sensor & B\&K4506 & 4 \\
\hline Synergy signal collection instrument & PH16H & 1 \\
\hline Power distribution box & XL-3-2 & 1 \\
\hline
\end{tabular}


In the gearbox's working process, the rotation speed is controlled by electromagnetic variable-speed motor, the load of magnetic-particle brake can be adjusted by the direct-current power to realize different rotation speed and different load. In the signal collection process, the sensor of B\&K4506 is located on the surface of gearbox, the sensor signals of vibration acceleration, rotation speed and rotation torque are recorded by data collection instrument of SynergyPH16H. The detailed composition of gearbox test table is shown as Table I.

Same time interval sampling method is adopted to collect the vibration response signals. The control device of electromagnetic variable-speed motor adjusts the rotation speed of gearbox. So the collection of vibration acceleration signals under different rotation speeds can be realized. In order to research the fault process of gears and bearings, the sensors are installed in the intermediate shaft, output shaft and bearing bases.

In the signal collection process, set the sampling frequency as $10 \mathrm{kHz}$, adjust the motor speed by the control device of electromagnetic variable-speed motor, measure the rotation speed of the gearbox's input shaft by rotation speed and torque sensors. The Synergy signal collection instrument has five channels. The CH1 channel is connected with JN338 rotation speed and torque sensor to collect the rotation speed signal of gearbox's input shaft. Other four channels are connected with four vibration acceleration sensor. The signal collection process should begin after the gearbox is working smoothly and steadily.

\section{TRADITIONAL SPECTRUAL KURTOSIS THEORY}

Spectral kurtosis theory was used to analyze the instantaneous impact composition in gear and bearing vibration signals firstly. Carry out Cramer decomposition to the signal $x(t)$. The corresponding expression is ${ }^{[3]}$

$$
X(t)=\int_{-\infty}^{+\infty} e^{j 2 \pi f t} H(t, f) d X(f)
$$

Where $H(t, f)$ is a complex envelope function. Adopt short time Fourier transform method to compute

$$
H(t, f)=\int_{-\infty}^{+\infty}[x(\tau) \gamma *(\tau-t)] e^{-j 2 \pi f t} d t
$$

Where $\gamma(\tau)$ is a window function with small time width. Define the fourth order spectrum cumulant of $Y(t)$ process is

$$
C_{4 Y}(f)=S_{4 Y}(f)-2 S_{2 Y}^{2}(f) \quad f \neq 0
$$

Where is the instantaneous torque spectrum of $2 \mathrm{n}$ orders.

$$
S_{2 n Y}(f)=E\left\{|H(t, f) d X(f)|^{2 n}\right\} / d f
$$

Then the spectral kurtosis is

$$
K_{Y}(f)=\frac{C_{4 Y}(f)}{S_{2 Y}^{2}(f)}=\frac{S_{4 Y}(f)}{S_{2 Y}^{2}(f)}-2
$$

If the signal $x(t)$ is stationary signal, the corresponding spectral kurtosis zero. If the signal $x(t)$ is interfered by stationary noise signal $b(t)$, its spectral kurtosis is ${ }^{[4]}$

$$
K_{Z}(f)=\frac{K_{X}(f)}{[1+\rho(f)]^{2}} \quad f \neq 0
$$

Where $\rho(f)$ is the bottom of signal to noise ratio of signal $x(t)$.

The Fourier transform has no effective treating method for the trade-off problem between the time domain and frequency domain resolution. The basis of spectral kurtosis is short time Fourier transform. So the spectral kurtosis method can not realize fault diagnosis under the condition of strong noise interference.

\section{IMPROVED SPECTRUAL KURTOSIS THEORY}

Energy operator is a kind of mathematical algorithm to track the narrowband signal. Define the energy operator of continuous signal $x(t)$ by mathematical method

$$
\Psi_{c}[x(t)]=\left(\frac{d x(t)}{d t}\right)^{2}-x(t) \frac{d^{2} x(t)}{d t^{2}}=[\dot{x(t)}]^{2}-x(t) \ddot{x}(t)
$$

Where $\Psi_{c}$ is the energy operator, $\dot{x(t)}$ and $\ddot{x}(t)$ is the first two derivative of the original signals.

As for an undamped free vibration system, the vibration displacement is

$$
x(t)=A \cos \left(\omega_{0} t+\theta\right)
$$

Its energy operator is

$$
\Psi_{c}[x(t)]=\Psi_{c}\left[A \cos \left(\omega_{0} t+\theta\right)\right]=\left(A \omega_{0}\right)^{2}
$$

The energy of undamped oscillation system has no change with time. And its energy value is equal to the sum of kinetic energy and potential energy at a certain moment. The computation formula is

$$
E_{0}=\left(A \omega_{0}\right)^{2} / 2
$$

As for discrete signal $x(n)$, the computation formula of energy operator is

$$
\Psi_{d}[x(n)]=x^{2}(n)-x(n-1) x(n+1)(n=0, \pm 1, \pm 2, \cdots)
$$


Define the sampling cycle of discrete signal as $T$, and replace the continuous variable $t$ by discrete variable $n T$. Establish the reflection between two kinds of signal energy operators as

$$
\left\{\begin{array}{l}
\dot{x(t)} \leftrightarrow[x(n)-x(n-1)] / T \\
\ddot{x}(t) \leftrightarrow[x(n)-2 x(n-1)+x(n-2)] / T^{2} \\
\Psi_{c}[x(t)] \leftrightarrow \Psi_{d}[x(n-1)] / T
\end{array}\right.
$$

\section{Gearbox Fault Detection and Diagnosis}

Because of the complex working condition and noise interference, fast spectral kurtosis algorithm can't extract the complete feature information. In this case, minimum entropy solution of convolution operation should be carried out to the original vibration signals of gearbox, and highlight the spike pulse composition. Fast spectral kurtosis algorithm is carried out to MED filtering signal again. Pass filter is finished under the optimal center frequency and bandwidth band. Energy operator demodulation method is adopted for envelope spectrum analysis of filtering signals. Compared with traditional Hilbert change method, energy operator demodulation method has high temporal resolution, high adaptive ability and simple computation process ${ }^{[2][3]}$. By comparing spectral envelope with fault frequency theory values of gearbox, different fault types can be analyzed.

In the gear tooth root crack fault experiment, the rotation speed of input shaft of gearbox is $1468.7 \mathrm{r} / \mathrm{min}$. According to the transmission ratio, the rotation speed of intermediate shaft can be calculated and is $738.35 \mathrm{r} / \mathrm{min}$. Furthmore, the rotation frequency of intermediate shaft and gear is

$$
f_{r}=12.24 H z
$$

And set the sampling interval is 2 s.

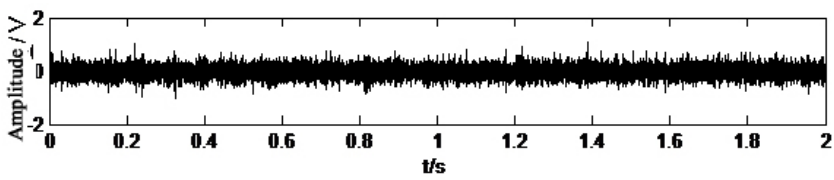

(A) TIME-DOMAIN FIGURE OF ROOT CRACK FAULT SIGNAL

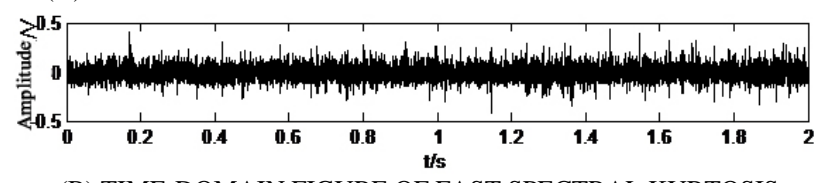

(B) TIME-DOMAIN FIGURE OF FAST SPECTRAL KURTOSIS FILTERING SIGNAL

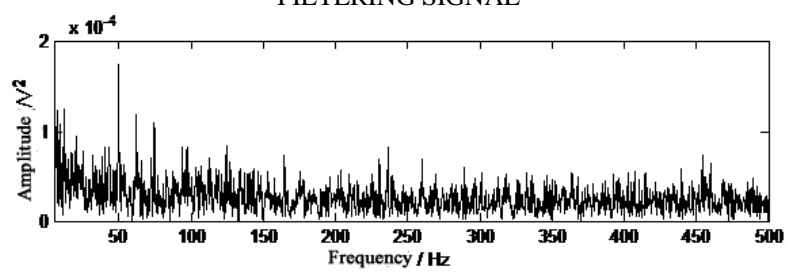

(C) SPECTRAL ENVELOPE FIGURE

FIGURE II. FAST SPECTRAL KURTOSIS FILTERING OF ROOT CRACK FAULT SIGNAL
Figure II is the time domain and spectral envelope of original root tooth crack fault signal after spectral kurtosis filtering. We can see from Figure II that although the impact properties are enhanced, the fault information also cannot be identified from the spectral envelope in Figure II (c).

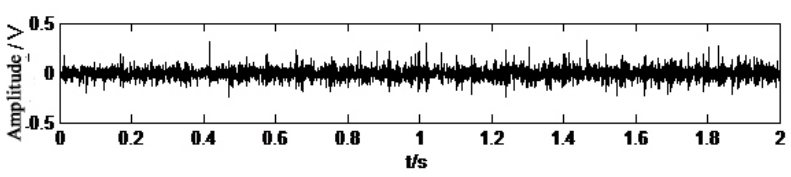

(A) MED TIME-DOMAIN FIGURE AFTER ORIGINAL SIGNAL NOISE REDUCTION

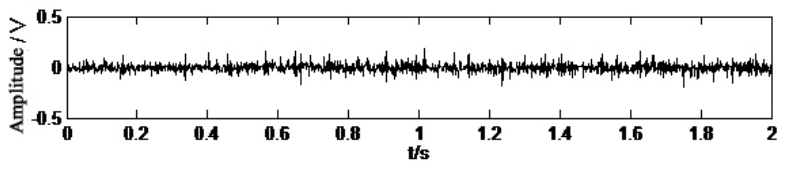

(B) FAST SPECTRAL KURTOSIS FILTERING FIGURE AFTER MED NOISE REDUCTION

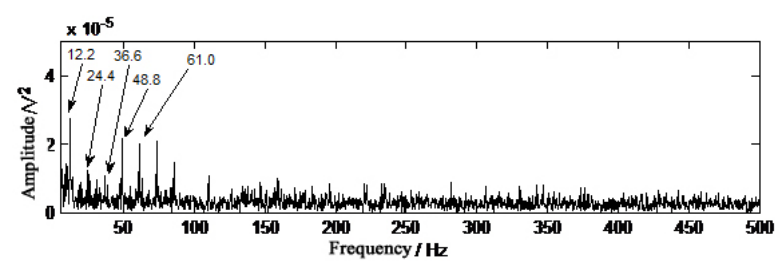

(C) SPECTRAL ENVELOPE FIGURE AFTER NOISE FILTERING

FIGURE III. FAST SPECTRAL KURTOSIS FILTERING AFTER MED NOISE REDUCTION

Figure III includes the MED time-domain figure after original signal noise reduction, fast spectral kurtosis filtering figure after MED noise reduction and spectral envelope figure after noise filtering.

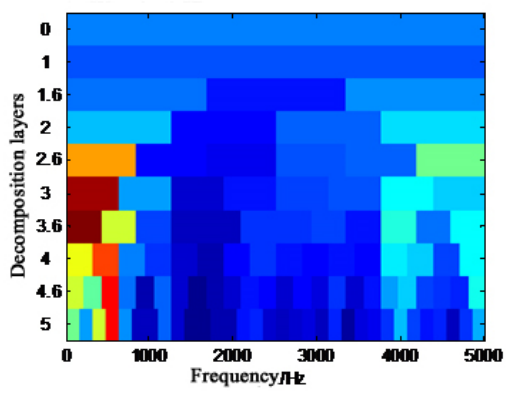

FIGURE IV. FAST SPECTRAL KURTOSIS SPECTRA AFTER MED NOISE REDUCTION

Figure IV is the fast spectral kurtosis spectra after MED noise reduction of fault signal in Figure III. Figure VI show that in fast spectral kurtosis spectra, the signal kurtosis value is maximum provided by band-pass filter when the decomposition layer is 3.6 , the central frequency is $208.33 \mathrm{~Hz}$, the bandwidth is $416.67 \mathrm{~Hz}$. The corresponding color in Figure VI is deepest. Then, the band-pass filter parameters can be calculated. In Figure III, after MED and fast spectral kurtosis filtering, the signal spectral envelope clearly identify the 12.2 $\mathrm{Hz}$ frequency part, which accords with formula (13) and verify the effectiveness of fault signal extraction method .

However, there are not only single faults, but also composite faults under the actual working condition, which 
require better fault detection and diagnosis method.

In composite crack fault diagnosis experiment of bearing's inner and outer rings, the rotation speed of input shaft of gearbox is $1492.3 \mathrm{r} / \mathrm{min}$, the rotation speed of intermediate shaft is $746.15 \mathrm{r} / \mathrm{min}$. Then, the fault frequency of bearing's inner ring is

$$
f_{\text {in }}=67.51 \mathrm{~Hz}
$$

The fault frequency of bearing's outer ring is

$$
f_{\text {out }}=44.54 \mathrm{~Hz}
$$

The rotation frequency of intermediate shaft is

$$
f_{r}=12.44 \mathrm{~Hz}
$$

And the sampling interval is 2s.

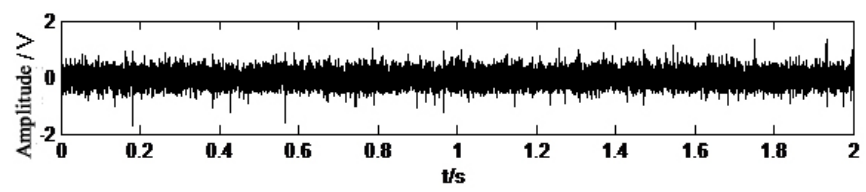

(A) TIME-DOMAIN FIGURE OF COMPOSITE CRACK FAULT ORIGINAL SIGNAL OF BEARING'S INNER AND OUTER RINGS

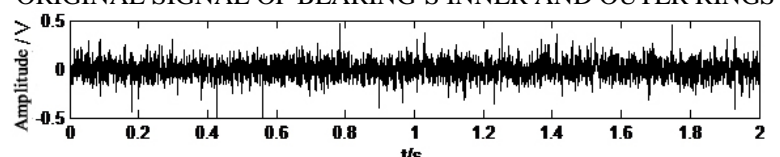

(B) MED SIGNAL AFTER NOISE REDCTION

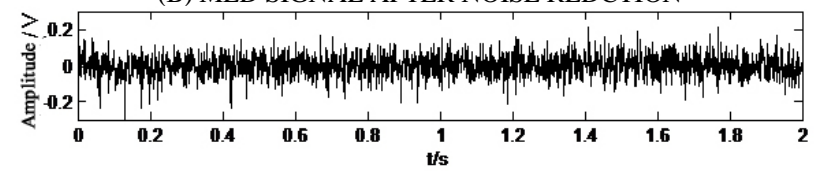

(C) TIME-DOMAIN FIGURE OF FAST SPECTRAL KURTOSIS FILTERING

FIGURE V. FILTERING SIGNAL FIGURE OF BEARING'S COMPOSITE FAULT

Figure $\mathrm{V}$ is the stepwise filtering graph of composite crack fault signal for bearing's inner and outer ring. In the timedomain figure of original signal, the shock response signal of crack fault is submerged by noise and is difficult to identify. After MED noise reduction and noise filtering of spectral kurtosis, the shock response characteristic is highlight. The comparison between signal's kurtosis values before and after noise filtering is shown in Table II. Kurtosis comparison of different filtering methods

TABLE II. KURTOSIS COMPARISON AMONG DifFERENT FILTERING METHODS

\begin{tabular}{|l|c|c|c|c|c|c|}
\hline Signal & $\begin{array}{c}\text { Original } \\
\text { Signal }\end{array}$ & FSK & MED & $\begin{array}{c}\text { MED+ } \\
\text { FSK }\end{array}$ & IMF3 & $\begin{array}{c}\text { High } \\
\text { Frequency of } \\
\text { Third Layer }\end{array}$ \\
\hline $\begin{array}{c}\text { Kurtosis } \\
\text { value }\end{array}$ & 1.16 & 2.88 & 16.25 & 18.42 & 1.38 & 1.21 \\
\hline
\end{tabular}

Table II shows that after MED and FSK filtering for original signal, the signal kurtosis value increase from 1.16 to 18.42, but the kurtosis value growth of other filtering methods is smaller. Figure VI is the signal spectral envelope after filtering.

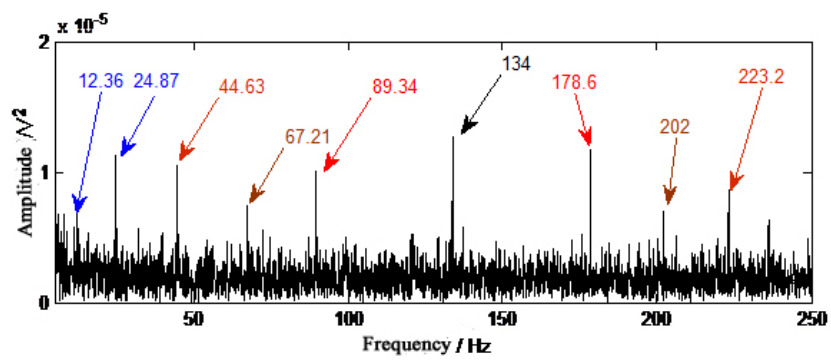

FIGURE VI. SIGNAL SPECTRAL ENVELOPE AFTER FILTERING

Figure VI shows that the signal's characteristic frequencies of MED filtering and FSK filtering are obvious. The frequency of $12.36 \mathrm{~Hz}$ accords with the rotation frequency of intermediate shaft. And the frequency of $24.87 \mathrm{~Hz}$ accords with the rotation frequency of input shaft. $44.63 \mathrm{~Hz}$ is the crack fault frequency of bearing's outer ring. $89.34 \mathrm{~Hz}, 134 \mathrm{~Hz}, 18.6 \mathrm{~Hz}$ and $223.2 \mathrm{~Hz}$ are according with the double frequency to five-times frequency of crack fault of bearing's outer ring. $67.21 \mathrm{~Hz}$ accords with the crack fault frequency of bearing's inner ring. $134 \mathrm{~Hz}$ and $202 \mathrm{~Hz}$ are according with the double frequency to three-times frequency of crack fault of bearing's inner ring. The above data verify the effectiveness of extraction method for composite fault feature extraction of bearing's inner and outer rings.

\section{CONCLUSION}

Aiming to the difficulties in fault signal feature extraction of traditional spectral kurtosis method under large noise working conditions, the minimum entropy convolution theory and spectral kurtosis algorithm are combined, and a new kind of gearbox fault diagnosis method based on MED and FSK is put forward. Single faults and composite faults on gearbox fault test table verifies the effectiveness and precision.

\section{REFERENCES}

[1] J. Antoni, "Fast Computation of the Kurtogram for the Detection of Transient Faults,” Mechanical Sysems and Signal Processing, vol. 21, pp. 108-124, October 2007.

[2] M. Pan, and W. Tsao, "Using Appropriate IMFs for Envelope Analysis in Multiple Fault Diagnosis of Ball Bearings,” International Journal of Mechanical Sciences, vol. 69, pp. 114-124, January 2013.

[3] R. H. Jiang, Sh. L. Liu, and Y. F. Tang, “A Novel Method of Fault Diagnosis for Rolling Element Bearings Based on the Accumulated Envelope Spectrum of the Wavelet Packed,” Journal of Vibration and Control, vol. 21, pp. 1580-1593, August 2015.

[4] J. Antoni, and R. B. Randall, "The Spectral Kurtosis: Application to the Vibratory Surveillance and Diagnostics of Rotatin Machines," Mechanical Systems and Signal Processing, vol. 20, pp. 308-331, February 2006. 